2011;18:271-5. http://dx.doi.org/10.1051/ parasite/2011183271

Address for correspondence: Attila D. Sándor, Department of Parasitology and Parasitic Diseases, University of Agricultural Sciences and Veterinary Medicine, Cluj-Napoca, Calea Mănăştur 3-5, RO-400372, Cluj, Romania; email: adsandor@gmail.com

\section{Serologic Survey of Plague in Animals, Western Iran}

To the Editor: Plague has been one of the most devastating infectious diseases in human history. The etiologic agent, Yersinia pestis, primarily affects rodents and is usually transmitted to humans through infective flea bites. Endemic plague foci result from circulation of the plague bacillus in its rodent reservoir, the source of human plague cases (1). Carnivores such as dogs and foxes, which prey on rodents and eat their fresh carcasses, are valuable sentinel animals for plague serosurveillance in disease-endemic foci, although their infections are usually asymptomatic $(2,3)$.

Plague epidemics have caused loss of human life in various parts of Iran. During 1947-1966 in western Iran, 9 human epidemics occurred and caused 156 deaths. The last case of human plague was reported in 1966 (4). Field investigations identified 4 Meriones rodent species as $Y$ pestis reservoirs; 2 were resistant (M. persicus and $M$. libycus), and the other 2 (M. tristrami and M. vinogradovi) were susceptible to death from infection $(4,5)$. The epidemiologic investigations demonstrated a 3-4 year plague epizootic cycle in Iran (5). The last official report of plague in rodents in Iran dates back to 1978 , in Sarab County in the East Azarbaijan Province (6). Plague surveillance was ignored for more than 3 decades and then restarted in 2011 in Iran.

This study was designed to investigate plague among resident animals in western Iran, specificallyregion localities along the border between the Kurdistan and Hamadan Provinces, where plague in wildlife has been repeatedly reported (enclosed by $47.900^{\circ}$ and $48.284^{\circ}$ north latitude and $35.4616^{\circ}$ and $35.7829^{\circ}$ east longitude). The epidemiologic team was based at the Akanlu Research Center of the Pasteur Institute of Iran, in a village $\approx 100 \mathrm{~km}$ from Kabudar Ahang, Hamadan Province, at an altitude of $\approx 1,600 \mathrm{~m}$. The study was conducted during June-September in 2011 and 2012. In 2011, a large area $\left(2,000 \mathrm{~km}^{2}\right)$ was selected and, because only $1 Y$. pestis-positive dog sample was found, in 2012 , , the study area was reduced to $1,200 \mathrm{~km}^{2}$ and confined to localities in which the $Y$. pestis-positive dog sample was identified the previous year; 3 additional $Y$. pestis-positive dogs and $1 Y$. pestis-positive rodent were found in 2012.

The average number of traps used per night per locality was 13 . A total of 46 rodents were entrapped from 26 localities in 998 traps $(4.61 \%$ success) during the first year, and 52 rodents were captured in 30 localities in 1,164 traps $(4.46 \%$ success $)$ during the second year. They were mostly members of the Meriones genus, although a few Microtus socialis irani and 1 Ellobius lutescens rodents were also caught (Table). A total of 281 fleas were collected on $70.41 \%$ of trapped rodents (Table), corresponding to an average flea index of 4.10 for infested rodents. All fleas were Xenopsylla spp. ELISA was performed as described (7) to detect antibodies against $Y$. pestis $\mathrm{F} 1$ capsular antigen. Samples positive by ELISA were confirmed by using the inhibition ELISA method (8). Of 98 trapped rodents, $1(1.02 \%)$ had IgG against F1 (Table), an M. persicus jird caught in 2012.
Sheepdogs that lived in the study areas were also used as sentinel animals. Blood samples were collected from 58 sheepdogs in 15 villages in 2011 and from 59 sheepdogs in 8 villages in 2012. Of $117 \mathrm{dog}$ serum samples analyzed, 4 (3.42\%) had IgG titers against F1, 1 in 2011 and the other 3 in 2012 (Table). Finally, wild animals such as jackals, foxes, rabbits, and hedgehogs were hunted in the study areas, and blood samples were taken immediately. None of the serum samples obtained from 3 foxes, 2 jackals, 8 rabbits, and 1 hedgehog had $\operatorname{IgG}$ against F1 (Table).

Because a well-established plague focus existed in Iranian Kurdistan, with animal cases occurring until 1978 (9), complete extinction of this focus is most unlikely. Our study demonstrates that animal reservoirs (Meriones rodents) and flea vectors (Xenopsylla spp.) shown to be central to the plague ecologic cycle in Iran still are found in high numbers in a previously active focus. The fact that $70 \%$ of trapped rodents were infested with fleas, with an average Xenopsylla spp. index of 4.10, may be considered as circumstances most favorable for the onset of plague epizootics. Furthermore, the detection of $Y$. pestis-specific IgG in $1.02 \%$ of trapped rodents and $3.42 \%$ of sentinel dogs is highly suggestive of active circulation of $Y$. pestis in its natural animal reservoir. Because $Y$. pestis antibodies last only for $\approx 6$ months in dogs (2), seropositivity of these dogs indicates newly acquired infections.

This fact that $Y$. pestis--positive animals were found over the 2-year surveillance period suggests that this area could be an active plague focus. Therefore, although no official reports of human plague in Iran have been made since 1966, this study indicates that the epidemiologic conditions needed to trigger an outbreak have been met. It is thus of utmost importance to maintain and strengthen the health system with plague surveillance in western Iran. 
Table. Number of fleas and serum samples with Yersinia pestis F1 antibody collected from various animals during field investigations, Iran, 2011-2012*

\begin{tabular}{|c|c|c|c|}
\hline Animal/species & No. serum samples & $\begin{array}{l}\text { No. serum samples with antibodies } \\
\text { against F1 }\end{array}$ & No. fleas collected \\
\hline \multicolumn{4}{|l|}{ Rodents } \\
\hline Meriones persicus & 39 & 0 & 33 \\
\hline Meriones vinogradovi & 3 & 0 & 0 \\
\hline Microtus socialis irani & 4 & 0 & 1 \\
\hline \multicolumn{4}{|l|}{2012} \\
\hline Meriones persicus & 24 & 1 & 146 \\
\hline Meriones libycus & 26 & 0 & 101 \\
\hline Microtus socialis irani & 1 & 0 & 0 \\
\hline Ellobius lutescens & 1 & 0 & 0 \\
\hline Total & 98 & 1 & 281 \\
\hline \multicolumn{4}{|l|}{ Sheepdogs } \\
\hline \multicolumn{4}{|l|}{2011} \\
\hline ND & 58 & 1 & ND \\
\hline \multicolumn{4}{|l|}{2012} \\
\hline ND & 59 & 3 & ND \\
\hline Total & 117 & 4 & \\
\hline \multicolumn{4}{|l|}{ Miscellaneous } \\
\hline \multicolumn{4}{|l|}{ Jackals } \\
\hline \multicolumn{4}{|l|}{2011} \\
\hline Canis aureus & 2 & 0 & ND \\
\hline \multicolumn{4}{|l|}{2012} \\
\hline C. aureus & 0 & 0 & ND \\
\hline \multicolumn{4}{|l|}{ Foxes } \\
\hline \multicolumn{4}{|l|}{2011} \\
\hline Vulpes vulpes & 1 & 0 & ND \\
\hline \multicolumn{4}{|l|}{2012} \\
\hline V. vulpes & 1 & 0 & ND \\
\hline \multicolumn{4}{|l|}{ Rabbits } \\
\hline \multicolumn{4}{|l|}{2011} \\
\hline Lepus capensis & 1 & 0 & ND \\
\hline \multicolumn{4}{|l|}{2012} \\
\hline \multirow{2}{*}{\multicolumn{4}{|c|}{ Hedgehogs }} \\
\hline & & & \\
\hline 2011 & 0 & 0 & \\
\hline ND & & & ND \\
\hline 2012 & 1 & 1 & \\
\hline ND & & & ND \\
\hline Total & 14 & 0 & \\
\hline
\end{tabular}

\section{Acknowledgments}

We appreciate the financial support of the Pasteur Institute of Iran and Center for Disease Control of the Iranian Ministry of Health and Medical Education (grant no. 582). We also express our gratitude to Mohammad Mehdi Goya and Mahmoud Soroosh for their scientific and logistic support; to Behzad Esfandiari, Hamed Hanifi, and Mohammad Hanifi, who helped us with sampling; and to Manijeh Yousefi-Behzadi, who assisted us with laboratory tasks.

\section{Saber Esamaeili, Kayhan Azadmanesh, Saied Reza Naddaf, Minoarisoa Rajerison,}

\section{Elisabeth Carniel, and Ehsan Mostafavi}

Author affiliations: Pasteur Institute of Iran, Tehran, Iran (S. Esamaeili, K. Azadmanesh, S.R. Naddaf, E. Mostsfavi); Institut Pasteur of Madagascar, Antananarivo, Madagascar (M. Rajerison); and Institut Pasteur, Paris, France (E. Carmiel)

DOI: http://dx/doi.org/10.3201/eid1909.121829

\section{References}

1. Stenseth NC, Atshabar BB, Begon M, Belmain SR, Bertherat E, Carniel E, et al. Plague: past, present, and future. PLoS Med. 2008;5:e3.. http://dx.doi. org/10.1371/journal.pmed.0050003

2. Brinkerhoff RJ, Collinge SK, Bai Y, Ray C. Are carnivores universally good sentinels of plague? Vector Borne Zoonotic Dis. 2009;9:491-7.. http://dx.doi. org/10.1089/vbz.2008.0075

3. Li B, Guo Y, Guo Z, Liang Y, Zhu Z, Zhou $Q$, et al. Serologic survey of the sentinel animals for plague surveillance and screening for complementary diagnostic markers to F1 antigen by protein microarray. Am J Trop Med Hyg. 2008;79:799-802.

4. Azizi MH, Azizi F. A history of the human plague in Iran. Arch Iran Med. 2010;13:563-9.

5. Baltazard M, Bahmanyar M, Mostachfi $\mathrm{P}$, Eftekhari M, Mofidi C. Recherches sur la peste en Iran. Bull World Health Organ. 1960;23:141-55.

6. Karimi Y, Mohammadi M, Hanif M. Methods for rapid laboratory diagnosis of plague and an introduction of a new plague foci in Sarab (East Azarbaijan). Journal of Medical Council of Islamic Republic of Iran. 1978;6:326-2. 
7. Rasoamanana B, Leroy F, Boisier $\mathrm{P}$, Rasolomaharo M, Buchy P, Carniel E, et al. Field evaluation of an immunoglobulin $\mathrm{G}$ anti-F1 enzyme-linked immunosorbent assay for serodiagnosis of human plague in Madagascar. Clin Diagn Lab Immunol. 1997;4:587-91.

8. Rajerison M, Dartevelle S, Ralafiarisoa LA, Bitam I, Tuyet DTN, Andrianivoarimanana $\mathrm{V}$ et al. Development and evaluation of two simple, rapid immunochromatographic tests for the detection of Yersinia pestis antibodies in humans and reservoirs.. PLoS Negl Trop Dis. 2009;3:e421.

9. Karimi Y. Discovery of a new intermediate focus of sylvatic plague in the eastern Azarbaijan region of Iran [in French]. Bull Soc Pathol Exot. 1980;73:28-35. PMID:7418121

Address for correspondence: Ehsan Mostafavi, No. 69, Pasteur Ave., Department of Epidemiology, Pasteur Institute of Iran, Postal Code: 1316943551, Tehran, Iran; email: mostafavi@pasteur.ac.ir

\section{Livestock Density as Risk Factor for Livestock- associated MRSA, the Netherlands}

To the Editor: We challenge the conclusions of Feingold et al. that "regional density of livestock is a notable risk factor for nasal carriage of LA-MRSA for persons with and without direct contact with livestock" (1). They did not study nasal carriage of methicillin-resistant Staphylococcus aureus (MRSA), but they retrospectively analyzed 87 culture-confirmed MRSA cases reported to a reference laboratory. These were a mixture of clinical disease isolates and screening (nose, throat, and perineum) isolates that were unevenly distributed between the groups (2). Because their analysis aimed to assess exposure risk by residential location, they should have excluded the 5 persons who acquired MRSA outside the Netherlands.

Table. Pig density in the Netherlands, United States (excluding Alaska), and major pigproducing states

\begin{tabular}{|c|c|c|c|c|}
\hline Location & No. pigs & Area, $\mathrm{km}^{2}$ & $\begin{array}{l}\text { Pig density, } \\
\text { pigs } / \mathrm{km}^{2}\end{array}$ & $\begin{array}{l}\text { Relative pig } \\
\text { density* }\end{array}$ \\
\hline The Netherlands & $12,100,000 \dagger$ & 41,518 & 291.4 & 1 \\
\hline United States & $67,500,000+$ & $8,108,782 \ddagger$ & 8.3 & 35.0 \\
\hline lowa & $19,700,000$ & 145,744 & 135.2 & 2.2 \\
\hline North Carolina & $8,600,000$ & 139,393 & 61.7 & 4.7 \\
\hline Minnesota & $7,600,000$ & 225,174 & 33.8 & 8.6 \\
\hline \multicolumn{5}{|c|}{$\begin{array}{l}\text { *Pig density in the Netherlands divided by pig density in other locations. } \\
\text { †US data were obtained from a quarterly US Department of Agriculture report } \\
\text { (http://usda01. library.cornell.edu/usda/nass/HogsPigs//2010s/2012/HogsPigs-09-28-2012.pdf). } \\
\ddagger \text { Alaska was excluded because of minimal swine industry. }\end{array}$} \\
\hline
\end{tabular}

Retrospective case-control studies preclude direct estimation of incidence, prevalence, or risk. However, because of the symmetric property of odds ratios, disease odds ratios can be inferred indirectly from the estimated exposure odds ratios in case-control studies (3). However, this case-case study design has no true controls, precluding valid inferences of absolute or relative risks. The higher ratio of livestock-associated (LA)-MRSA to a typeable strain of MRSA (T-MRSA) in rural cases could be attributable to higher risk for LA-MRSA in rural areas, lower risk for T-MRSA in rural areas, or both.

To illustrate this point, suppose urban dwellers had equal prevalence rates of LA-MRSA and T-MRSA of $5 \%$, and rural dwellers had prevalence rates of $2 \%$ for LA-MRSA and $1 \%$ for T-MRSA. The ratio approach used would indicate that rural dwellers had twice the risk for LA-MRSA than urban dwellers, when the absolute risk is 2.5 times higher in the urban group. At best, their conclusion could be viewed as a hypothesis that should be tested.

Three large community-based studies with better methods collectively refute this hypothesis. Across these studies, LA-MRSA prevalence (44\%) was $>180$ times higher in 352 occupationally exposed persons than in 2,094 rural residents without farm exposure (0.24\%) (4-6). Prevalence in family members of livestock workers was intermediate $(5.2 \%)$. These consistent observations indicate that exposure to LA-MRSA in livestock-dense regions is a common occupational risk for livestock workers, a lesser indirect risk to their family members, and a negligible risk to persons without livestock or farm contact.

Finally, the contention of Feingold et al. that pig production in the Netherlands is "greatly overshadowed by the density of pig-farming operations in the United States" is mistaken (1) (Table). Pig density in the Netherlands is 35 times higher than in the United States, and more than twice that in Iowa.

\section{Peter R. Davies, Bruce H. Alexander, Jeffrey B. Bender, John Deen, Catherine E. Dewey, Julie A. Funk, Claudia A. Munoz-Zanzi, M. Gerard O'Sullivan, Randall S. Singer, Srinand Sreevatsan, Katharina D. Stärk, and Mark A. Stevenson}

Author affiliations: University of Minnesota College of Veterinary Medicine, St. Paul, Minnesota, USA (P.R. Davies, J.B. Bender, J. Deen, M.G. O'Sullivan, R.S Singer, S. Sreevatsan); University of Minnesota School of Public Health, Minneapolis, Minnesota, USA (B.H. Alexander, C. A. MunozZanzi); Ontario Veterinary College, Guelph, Ontario, Canada (C.E. Dewey); Michigan State University College of Veterinary Medicine, East Lansing, Michigan, USA (J.A. Funk); Royal Veterinary College, London, United Kingdom (K.D. Stärk); and Massey University, Palmerston North, New Zealand (M.A. Stevenson)

DOI: http://dx.doi.org/10.3201/eid1909.121577 